\title{
A TRANSFER MATRIX METHOD FOR STUDY OF VibRations of Plane Bars Systems
}

\author{
BoiAngiU, M. \& AleCU, A.
}

Abstract: In this paper we study the simultaneous vibrations of bars systems, considering as been continuous medium. We obtained for the transfer matrix method because the finite elements method introduces specific features. We divide the system in cycles and we apply the transfer matrix method associated with transit matrix method for each cycle. We develop the free vibrations cases. We apply the purposed method to the concrete cases of a statically determinate system and of two statically indeterminate systems. The method can be applied associated with the graph theory, for identifying the cycles and the types of nodes. The method is indicated to be used in computational engineering, especially for the complicated bars systems.

Key words: vibrations, matrices, frequencies, vectors, cycles
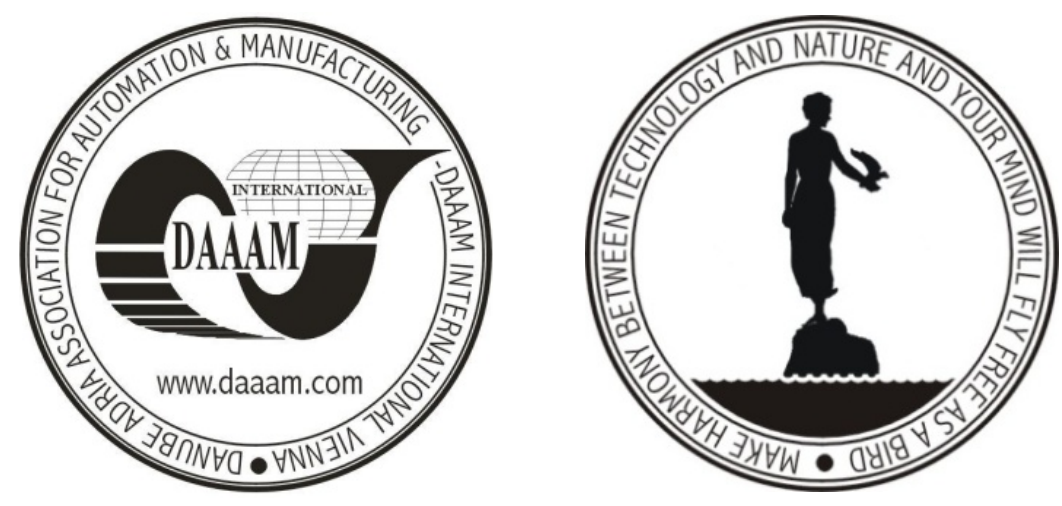

Author's data: Univ. Lecturer. Dipl.-Eng. Dr.techn. Boiangiu, M[ihail]; Univ. Lecturer. Dipl.-Eng. Dr.techn. Alecu, A[urel], "Politechnica” University of Bucharest, Department of Mechanics, Splaiul Independentei, no.313, 060042, Bucharest, Romania, mboiangiu@gmail.com, aurel_alecu@yahoo.com

This Publication has to be referred as: Boiangiu, M[ihail] \& Alecu, A[urel] (2009). A transfer matrix method for study of vibrations of plane bars systems, Chapter 77 in DAAAM International Scientific Book 2009, pp. 798-814, B. Katalinic (Ed.), Published by DAAAM International, ISBN 978-3-901509-69-8, ISSN 1726-9687, Vienna, Austria

DOI: $10.2507 /$ daaam.scibook.2009.77 